\title{
Physical Activity and Mental Health in Young People
}

\author{
Stuart J.H. Biddle \\ University of Southern Queensland \\ Institute for Resilient Regions \\ Springfield, QLD, Australia \\ stuart.biddle@usq.edu.au
}

\begin{abstract}
This paper reviews the latest evidence on whether physical activity for children and adolescents is associated with depression, anxiety, self-esteem, and cognitive functioning. Results from an updated review of systematic reviews are presented and discussed. It is concluded that physical activity is causally associated with cognitive functioning, partially so with depression, but not with self-esteem. Evidence is limited concerning anxiety.
\end{abstract}

Keywords-anxiety, cognitive function, depression, selfesteem, causality

\section{INTRODUCTION}

Physical activity has multiple health benefits for young people, and that is probably a 'given' in research, professional practice, and lay understanding. But the mental health of many young people in high income countries is not optimal and thus provides an important justification for promoting physical activity. In Australia, for example, 200809 data show 1.2 million mental health-related general practice encounters for young people (aged 16-24 years) annually, and this has been increasing. The second National Survey of the Mental Health and Wellbeing of Australian Children and Adolescents (2013-14) reported that a mental disorder was experienced by $14 \%$ of children and adolescents aged 4-17 years, and included major depressive and anxiety disorders [1].

It is important to note that even in an early academic paper Layman [2] stated that the psychological benefits of physical activity had been "a part of the literature ... for over 2000 years" but that claims "were often quite extravagant, without the benefit of supporting scientific evidence" (p. 33) [3]. It is common to see claims that physical activity in essentially 'good' for young people without necessarily recognising that positive mental health benefits may depend on the experience of physical activity and the context it takes place in rather than being an automatic benefit of engagement in any physical activity.

Typically, mental health has been defined quite broadly. For example, the World Health Organisation (WHO) defines it as "a state of well-being in which the individual realizes his or her own abilities, can cope with the normal stresses of life, can work productively and fruitfully, and is able to make a contribution to his or her community". It is not just about the alleviation of poor mental health, for example. Indeed, there has been a trend to give greater recognition to 'positive psychology' in recent years and to recognise the importance of such factors as positive self-esteem, mastery, personal growth, and resilience. However, as far as research trends go, the outcomes of depression, anxiety, self-esteem, and cognitive functioning have accumulated a substantial evidence base and will comprise the focus of this paper.

\section{UPDATING OUR EVIDENCE}

\section{A. A Review of Reviews}

We reviewed the evidence linking involvement in physical activity with mental health in young people [4]. Specifically, we conducted a review of reviews on the outcomes of depression, anxiety, self-esteem, and cognitive functioning. The paper has had over 400 citations on Scopus and over 800 on Google Scholar, and has a Field-Weighted Citation Impact score of 11.91 at September, 2018. What did we conclude in 2011?

- Depression: from four systematic reviews it was concluded that "physical activity over no intervention seems to be potentially beneficial for reduced depression, but the evidence base is limited" (p. 888).

- Anxiety: from four systematic reviews it was concluded that "physical activity interventions for young people have been shown to have a small beneficial effect for reduced anxiety. However, the evidence is limited and in need of development" (pp. 888-889).

- Self-esteem: from three systematic reviews we concluded that "physical activity can lead to improvements in self-esteem. However, there is a paucity of good quality research" (p. 889).

- Cognitive functioning: from seven systematic reviews, including one narrative review, we concluded that "routine physical activity can be associated with improved cognitive performance, classroom behaviour and academic achievement in young people, but these associations are usually small and not entirely consistent" (p. 894).

These findings suggest that physical activity has promise for the promotion of better mental health in young people, at least for the four outcomes assessed, but the results are still somewhat mixed and reflective of low quality research designs. Also, given that interest in young people's mental health seems to have increased, and the field of systematic reviews has expanded greatly in recent years, we felt it was 
timely to see if the conclusions reported by Biddle and Asare were still appropriate some 6-7 years later.

We have recently conducted an updated review of reviews of the Biddle and Asare paper, and in this we assessed whether any association between physical activity and mental health outcome can be considered causal [5]. Specifically, we assessed some of the criteria proposed by Sir Austin Bradford in his classic paper on causality in epidemiological research [6]. Our update included searches for systematic reviews between November 2010 and the end of 2017.

\section{B. Mental Health Outcomes}

In our analysis of depression, we located 10 new systematic reviews of mainly healthy individuals. Seven reviews addressed evidence from interventions and found that effect sizes ranging from -0.41 to -0.61 (considered 'moderate' in size), although these were slightly higher when samples were depressed.

For anxiety, only three new reviews were located. Effects were highly variable, and it was concluded that the literature remains 'small and fragmented'.

For self-esteem, 10 new reviews were found, again with largely healthy samples. Given the complexity of self-esteem as a psychological construct, it is not surprising that our results were mixed. Effect sizes from intervention studies ranged from 0.12 to 0.78 , and across all studies there was a variety of physical activity provision, including leisure-time physical activity, physical education, sport and fitness, and dance. However, clear associations for self-esteem were found in a review of resistance training activities.

For cognitive functioning, 25 new systematic reviews were located over the 7 -year period. These reviews included over 350 studies. It is clear that this field has expanded greatly in recent years. Cognitive functioning can best be assessed through cognitive tests, usually in a lab, or brain structure and function assessments using imaging techniques. In addition, markers can be ascertained from academic achievement scores. Regarding cognitive function, meta-analytic effect sizes for those without cognitive impairment were small but significant (0.20-0.43). Larger effects were shown for those with ADHD from 6-10 weeks of aerobic exercise (0.58-0.84). The most comprehensive systematic review was reported by Donnelly et al. [7] and this is published as a Position Stand paper of the American College of Sports Medicine. For cognitive outcomes, they concluded that children with higher fitness showed better cognitive performance and this was across longitudinal and cross-sectional studies. They also concluded that interventions showed improvements in executive function tests from physical activity programs. In summary, this evidence synthesis showed that positive cognitive outcomes can arise from physical activity and physical fitness.

Donnelly et al. [7] were the only researchers to systematically review the effects of physical activity and fitness on brain structure (e.g., neural architecture) and function (e.g., fMRI). They concluded that physical activity and aerobic fitness were beneficial for neural networks supportive of executive functioning.
Results concerning academic achievement were a little less clear. Effect sizes tended to be smaller than for cognitive function tests. Donnelly et al. [7] concluded positive associations between physical fitness and academic achievement but more mixed findings for physical activity interventions. Clearly linking activity or fitness to academic outcomes is likely to be difficult as academic achievement will be influenced by main factors.

\section{Can these Associations be Considered Causal?}

Each mental health outcome was assessed for evidence regarding causality. The following criteria were used: strength of association, consistency, temporal sequencing, coherence and biological plausibility, dose-response relationship, and experimental evidence. These are key indicators proposed for epidemiology research by Hill [6] otherwise known as the 'Bradford Hill criteria'. The key questions being addressed here are:

- Strength of association: how strong is the association between physical activity and the mental health outcome of interest?

- Consistency: how consistent is the evidence across different populations and in different settings?

- Temporal sequencing: does physical inactivity precede a change in mental health?

- Coherence and biological plausibility: does interpretation of the data remain broadly consistent with what is known about mental health, and is there biological plausibility linking physical activity and the mental health outcome of interest?

- Dose-response relationship: do higher levels of physical activity show better levels of mental health?

- Experimental evidence: is there experimental evidence for better mental health from changes in physical activity?

TABLE I. Summary ASSESSMENTS WhETHER PHySICAL ACTIVITY IS Causality Associated With Mental Health In Young People

\begin{tabular}{|c|c|c|c|}
\hline Criterion & \multicolumn{3}{|c|}{ Evidence for Causality } \\
\hline $\begin{array}{c}\text { Strength of } \\
\text { association }\end{array}$ & Pepression & Self-Esteem & $\begin{array}{c}\text { Cognitive } \\
\text { Function }\end{array}$ \\
\hline $\begin{array}{c}\text { Consistency } \\
\text { Temporal } \\
\text { sequencing }\end{array}$ & Partial & Partial & Yes \\
\hline $\begin{array}{c}\text { Coherence and } \\
\text { biological } \\
\text { plausibility }\end{array}$ & No & No & Partial \\
\hline $\begin{array}{c}\text { Dose-response } \\
\text { relationship }\end{array}$ & No & Partial & Yes \\
\hline $\begin{array}{c}\text { Experimental } \\
\text { evidence }\end{array}$ & Yes & Yo & No \\
\hline $\begin{array}{c}\text { Overall } \\
\text { appraisal }\end{array}$ & Partial & No & Yes \\
\hline
\end{tabular}


Table 1 summarises our appraisal for depression, selfesteem, and cognitive functioning based on our updated review of reviews. We did not consider the evidence extensive enough to provide a judgement for anxiety. Overall, we concluded physical activity was causally associated with cognitive functioning, partially so for depression, but not for self-esteem. The latter is highly complex and further research is required.

\section{CONCLUSIONS AND COMMENTARY}

One purpose of our review of reviews was to update from our 2011 review and see what has changed. Our conclusions since 2011 have changed somewhat. In 2011 we said that the effects for self-esteem seemed to be the strongest, but this is no longer the case. A significant increase in the quantity and quality of evidence regarding cognitive functioning and, to a lesser extent, depression, now shows these two outcomes to be more clearly associated with physical activity than self-esteem. However, selfesteem is a particularly complex area.

Across the three mental health outcomes of depression, self-esteem, and cognitive functioning where we were able to assess for causality (see Table 1), strength of association was evident, albeit with more variability for depression and self-esteem. But the general lack of support for temporal sequencing (i.e., physical activity preceding the mental health outcome measure) is a weakness. Similarly, there is no evidence across these three mental health outcomes for a dose-response relationship. While this is also a weakness, there may be more complex associations between dose of physical activity and outcomes than are currently assessed. For example, the association may be linear, curvilinear, or contain a threshold, after which no further gains in mental health are made. At this stage, we cannot conclude on any of these due to the lack of evidence. Moreover, 'dose' can be defined in several ways, including physical activity intensity, frequency, and duration. All three outcomes assessed for causality showed support from experimental evidence. This alone provides some confidence that physical activity has mental health effects.

There were somewhat mixed findings regarding depression and this could be due to the diversity of sampling of young people, including being 'healthy', having mild depressive moods, with clinical levels of depression, and also including other conditions (e.g., ADHD). Where the sampling was more focused on those with depression, results clearly favoured physical activity.

Much more is needed concerning chronic studies of anxiety and stress reduction, particularly during periods of prolonged stress, such as for examinations. Moreover, selfesteem results showed a somewhat mixed picture. Causality was not supported, although there was partial support for strength of association and coherence, and support from experimental evidence. However, self-esteem is a complex field and is replete with definitional and conceptual ambiguity. Some studies focus only on global self-esteem and ignore arguably more relevant sub-domains of selfesteem, such as physical self-worth.
Results from the reviews addressing physical activity and cognitive functioning showed the strongest evidence for causality. One possible explanation for cognitive effects of physical activity is through the effect on executive functioning which de Greef et al.[8] define as "higher order cognitive functions that are responsible for initiating, adapting, regulating, monitoring, and controlling information processes and behaviour" (p. 501).

The changes in cognitive and neuro-biological measures from physical activity might logically lead to enhanced academic performance. However, research on physical activity and academic performance is a complex field replete with biases and poor measures. For example, studies using teacher assessments can be non-blinded and biased, and some measures may not be appropriate and open to biases from the social and cultural context. This probably accounts for the mixed effects of physical activity reported in our current omnibus review. That said, if stronger effects can be shown, this will have major implications for the important role of physical activity in schools. For example, emerging evidence is available on the role of more active classrooms, but more is needed on whether physical activity breaks can be effective for learning and performance [7].

There is much to do in creating strong research designs in this field, but analysing data from the perspective of reviewing existing reviews, the field does appear to be improving, and evidence does show associations across mutliple mental health outcomes. Future research is needed to confirm our appraisals of causality.

\section{REFERENCES}

[1] D. Lawrence, et al. "The mental health of children and adolescents: report on the second Australian Child and Adolescent Survey of Mental Health and Wellbeing". Canberra: Department of Health, 2015.

[2] E. M. Layman, "Psychological effects of physical activity". Exercise and Sport Sciences Reviews, 1974, Vol 2, pp. 33-70.

[3] S. J. H. Biddle, I. Vergeer. "A brief history of exercise psychology". In Handbook of sport and exercise psychology: Vol 2 - Exercise psychology, M. Anshell and S. Petruzzello, Eds. Washington, DC: American Psychological Association, in press.

[4] S. J. H. Biddle, M. Asare. "Physical activity and mental health in children and adolescents: A review of reviews". British Journal of Sports Medicine, 2011, Vol 45, pp. 886-895.

[5] S. J. H. Biddle, S. Ciaccioni, G. Thomas and I. Vergeer. "Physical activity and mental health in children and adolescents: an updated review of reviews and an analysis of causality. Psychology of Sport and Exercise, in press.

[6] A. B. Hill, "The environment and disease: Association or causation?" Proceedings of the Royal Society of Medicine, 1965, Vol 58(5), pp. 295-300.

[7] J. E. Donnelly, et al. "Physical activity, fitness, cognitive function, and academic achievement in children: a systematic review". Medicine and Science in Sports and Exercise, 2016, Vol 48(6), pp. 1197-1222.

[8] J. W. de Greeff, R.J. Bosker, J. Oosterlaan, C. Visscher, and E. Hartman. "Effects of physical activity on executive functions, attention and academic performance in preadolescent children: a metaanalysis". Journal of Science and Medicine in Sport, 2018, Vol 21(5), pp. 501-507. 